\title{
Exploring the electrophysiological correlates of the default-mode network with intracerebral EEG
}

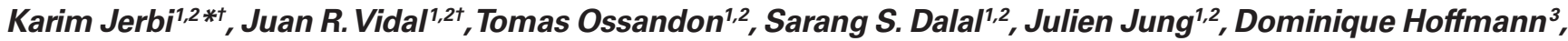 Lorella Minotti ${ }^{3}$, Olivier Bertrand ${ }^{1,2}$, Philippe Kahane ${ }^{3}$ and Jean-Philippe Lachaux ${ }^{1,2}$}

1 Institut National de la Santé et de la Recherche Médicale, U821, Brain Dynamics and Cognition, Lyon, France

2 Université Claude Bernard, Lyon 1, Lyon, France

${ }^{3}$ Neurology Department, Grenoble Hospital, Grenoble, France

\section{Edited by:}

Lucina Q. Uddin, Stanford University, USA

\section{Reviewed by:}

Biyu J. He, Washington University

School of Medicine, USA

Helmut Laufs, Johann Wolfgang

Goethe-University, Germany

*Correspondence:

Karim Jerbi, Institut National de la Santé et de la Recherche Médicale, U821, Brain Dynamics and Cognition, Centre Hospitalier Le Vinatier, Bâtiment 452, 95 Boulevard Pinel, 69500 Lyon,

France.

e-mail: karim.jerbi@inserm.fr

${ }^{+}$Karim Jerbi and Juan R. Vidal have contributed equally to this work.
While functional imaging studies allow for a precise spatial characterization of resting state networks, their neural correlates and thereby their fine-scale temporal dynamics remain elusive. A full understanding of the mechanisms at play requires input from electrophysiological studies. Here, we discuss human and non-human primate electrophysiological data that explore the neural correlates of the default-mode network. Beyond the promising findings obtained with non-invasive approaches, emerging evidence suggests that invasive recordings in humans will be crucial in order to elucidate the neural correlates of the brain's default-mode function. In particular, we contend that stereotactic-electroencephalography, which consists of implanting multiple depth electrodes for pre-surgical evaluation in drug-resistant epilepsy, is particularly suited for this endeavor. We support this view by providing rare data from depth recordings in human posterior cingulate cortex and medial prefrontal cortex that show transient neural deactivation during task-engagement.

Keywords: default-mode network, electrophysiology, gamma-band activity, stereotactic-electroencephalography, intracranial EEG

\section{INTRODUCTION}

The fact that parts of our brain are active even when we are not overtly engaged with the external world may not appear to be that much of a surprise per se. The fact that thoughts and inner mental processes are ongoing, and that they are certainly more prominent when we are not processing stimuli from the outside world, makes the concept of ongoing brain activity not only plausible but crucial. By contrast, what is definitely striking is the significant discrepancy between how much we have learned about the spatial characteristics of the so-called "default-mode" of brain function (Raichle et al., 2001) and how little we know about the precise neural mechanisms underlying its modulations and the fine-scale temporal dynamics thereof.

Over recent years, the default-mode network (DMN) (Gusnard and Raichle, 2001; Raichle et al., 2001) has been examined in the light of its putative relationship to self-cognition (Gusnard et al., 2001) and mind wandering (Mason et al., 2007). Deactivation of the DMN has been implicated in attention and task-engagement (Corbetta and Shulman, 2002) and its dysfunction has been linked to various mental disorders (Greicius, 2008; Broyd et al., 2009). A steady flow of seminal findings advancing our understanding of intrinsic brain activity continues to emerge from neuroimaging studies. Current important topics include the use of functional magnetic resonance imaging (fMRI) to investigate intrinsic network dynamics and connectivity patterns (Greicius et al., 2003; Fox et al., 2005; Uddin et al., 2009) and the putative relationship between DMN deactivations and behavioral performance (Weissman et al., 2006; Shulman et al., 2007; Anticevic et al., 2010). Nevertheless, the quest to fully elucidate the function of intrinsic brain networks also requires a solid understanding of the link between neuroimaging findings and their electrophysiological underpinnings.

In this paper we provide perspectives on the necessity, feasibility, and limitations of tackling the electrophysiological properties of DMN dynamics. We will discuss the utility and limitations of non-invasive electrophysiological techniques such as electroencephalography (EEG) and magnetoencephalography (MEG) in this endeavor. Most importantly, we will focus on the potential of direct electrophysiological recordings in humans to unravel the spectral and temporal properties of task-related changes of population activity in DMN structures.

A parallel stream of research in humans has revealed that bloodoxygenation level-dependent (BOLD) signal increases are tightly coupled with task-related power increases in the high-frequency range (broad-band gamma, $50-150 \mathrm{~Hz}$ ) of the intracranial EEG signal (Mukamel et al., 2005; Lachaux et al., 2007a; Nir et al., 2007). It is therefore tempting to ask whether task-related BOLD deactivations, typical for DMN areas, are in turn associated with suppressions of high gamma power. While human studies of gamma power increases are abundant (e.g., Lachaux et al., 2005; Crone et al., 2006; Jensen et al., 2007; Jerbi et al., 2009a), little is known about task-related gamma power suppression. Intracerebral studies from our group were the first to provide direct evidence in humans for task-related decreases of broad-band gamma $(>50 \mathrm{~Hz})$ power during performance of attention-demanding cognitive tasks (Lachaux et al., 2005, 2008). More recent studies (Mainy et al., 2008; Miller et al., 2009; Jung et al., 2010) provide further evidence for the co-occurrence of 
task-related increases and decreases of broad-band gamma activity in distinct brain areas during goal-directed behavior. As a matter of fact, because direct recordings from the cortex are not affected by physiological noise (e.g., breathing or cardiac changes), such studies are critical to refute claims that DMN observations constitute an epiphenomenon not of neuronal origin (Birn et al., 2008). Besides, we argue that depth recordings in humans will be key to probing the temporal properties of DMN deactivation and to unraveling the role of gamma activity therein. To further support this claim, we present rare human intracerebral stereotactic-EEG (SEEG) data recorded directly from two prominent DMN areas, namely the posterior cingulate cortex (PCC) and the medial prefrontal cortex (MPFC). Using time-frequency analysis we computed temporal and spectral profiles of population-level activity depicting task-related gamma-band deactivation in these areas during performance of attention-demanding tasks. Finally, we discuss some implications of our findings and we outline directions for future research in this challenging and rapidly growing field.

\section{INVESTIGATING DMN WITH ANIMAL ELECTROPHYSIOLOGY}

Unfortunately, our knowledge of the neural correlates of DMN remains elusive. This is in part due to the fact that investigating the electrophysiological correlates of the BOLD signal is a technically challenging endeavor and acquiring electrophysiological signals from human DMN structures faces multiple challenges. So what have electrophysiological approaches taught us about the neural correlates of DMN and what are their current limitations? Let us address this question first of all from the perspective of animal studies. A highly interesting study by Hayden et al. (2009) has reported significant task-related suppression of neuronal firing rate in macaque PCC a region considered to be a prominent component of DMN. As in previous reports of task-related BOLD deactivation, the reduction in neuronal firing in macaque PCC occurred during task performance and was followed by a return to higher baseline levels between trials. Most importantly, the firingrate suppression reported by Hayden et al. (2009) was predictive of performance (errors and reaction times). Despite the fact that the BOLD signal was not recorded in this study, the authors argue that the relationship to fMRI findings is strengthened by the fact that the activity of lateral intraparietal (LIP) neurons was enhanced during the task, i.e., LIP showed the inverse effect observed in PCC. Such non-human primate studies hold the potential to advance our understanding of the neural correlates of the DMN. The degree to which animal data can be generalized to humans may be restricted by the limits of anatomo-functional cross-species comparison. However, a more serious limitation to the study of DMN function with animal recordings arises if we want to test specific hypotheses about its putative role in mediating internally oriented mental processes (e.g., self-cognition, episodic and prospective memory, covert speech, etc.). Nevertheless, by contrast to electrophysiology, imaging studies in anesthetized animal can provide insights into the large-scale functional architecture of the DMN. As a matter of fact, the detection of spontaneous BOLD correlations (typical of resting state networks) in anesthetized monkeys (Vincent et al., 2007) has direct implications on the ongoing debate on the correlations between DMN connectivity and levels of consciousness (Greicius et al., 2008).

\section{NON-INVASIVE INVESTIGATION OF DMN WITH EEG}

Non-invasive electrophysiological techniques such as EEG or MEG provide whole-head coverage at a high temporal (millisecond-range) resolution and thus carry the potential to unravel the fine-temporal dynamics of the brain's intrinsic activity. Several EEG studies suggest various relationships between resting state networks and multiple spatial and spectral properties of the EEG. In particular, combining EEG and fMRI recordings provides a powerful framework for the comparison between various electrophysiological components and the BOLD responses during resting states (Laufs et al., 2003; Debener et al., 2005; Mantini et al., 2007; Laufs, 2008; Scheeringa et al., 2008; Jann et al., 2009). A first step toward assessing the EEG correlates of DMN is to decipher the way non-invasive surface measurements relate to the BOLD response. This question has been addressed by correlating BOLD with EEG power in various frequency bands. For instance, the BOLD signal has been shown to correlate negatively with EEG power in the alpha band (Goldman et al., 2002; Moosmann et al., 2003) and a recent study found positive correlations between BOLD and MEG high gamma power (Zumer et al., 2010). Numerous studies found correlations between DMN activity patterns and the power in traditional EEG frequency bands including theta $(4-7 \mathrm{~Hz})$, alpha $(8-12 \mathrm{~Hz})$, beta $(13-30 \mathrm{~Hz})$, and low-gamma (30-50 Hz) bands (Laufs et al., 2003; Mantini et al., 2007; Chen et al., 2008; Scheeringa et al., 2008; Jann et al., 2009). In contrast, putative links between BOLD responses and components in the lower end of the EEG frequency spectrum, namely delta oscillations (1-4 Hz), slow cortical potentials (SCPs), and infra-slow fluctuations $(0.01-0.1 \mathrm{~Hz})$ have proven harder to establish (Khader et al., 2008). Infra-slow EEG fluctuations (e.g., Monto et al., 2008) and SCPs have been proposed to reflect slow fluctuations in fMRI spontaneous activity (He and Raichle, 2009).

More generally, attempts to use non-invasive electrophysiological methods such as EEG or MEG to elucidate the neural mechanisms of intrinsic brain networks are challenged by two main limitations: the poor spatial resolution of MEG/EEG and the relatively limited signal-to-noise ratio of surface measurements especially with regards to detecting higher frequency components of the signal, namely the high gamma-band $(\sim 60-200 \mathrm{~Hz})$. Advanced MEG/EEG source reconstruction techniques yield cortical activation maps that are physiologically easier to interpret than sensor-level topographies (e.g., Baillet et al., 2001; Dalal et al., 2008). Nevertheless, the estimation of deeper sources in MEG/EEG is less reliable than the localization of activity from sources close to the sensors. This could be a severe limitation when it comes to detecting activity from deep regions of the default-mode such as the PCC. Moreover, the fact that high-frequency activity in the gamma-range is less easily detected with surface recordings (Pfurtscheller and Cooper, 1975; Jerbi et al., 2009a) might also be considered a further obstacle in this endeavor. As mentioned earlier, high gamma activity is an important target signal for DMN investigations because of its putative coupling with the BOLD signal (Logothetis et al., 2001; Niessing et al., 2005; Nir et al., 2007; Lachaux et al., 2008). Recently a number of studies have shown that MEG and EEG can, under certain circumstances, be used to detect task-related activity above $60 \mathrm{~Hz}$ (e.g., Ball et al., 2008; Cheyne et al., 2008; Dalal et al., 2008, 2009; Tecchio et al., 2008; Waldert et al., 2008; Van Der Werf et al., 2010; Zumer et al., 2010). 


\section{INTRACEREBRAL RECORDINGS: CLINICAL SETTING AND TECHNICAL FEATURES}

Fortunately, access to high resolution spatial and temporal signals through direct recordings from the human brain is sometimes possible in some clinical settings. Various types of invasive recordings from cortical and subcortical structures are used in conjunction with several clinical conditions (Engel et al., 2005). The surgical treatment of drug-resistant epilepsy requires intracranial recordings in multiple brain areas in order to localize the epileptic tissue (Kahane et al., 2004, 2006). During this pre-surgical evaluation period, electrical cortical stimulation and task-related functional mapping (Crone et al., 2006; Jerbi et al., 2009a) are used to map out healthy and eloquent cortex that should be spared during surgery. The two main invasive recording techniques used in the field of epilepsy consist of grid electrode placement over the cortex, a procedure known as Electrocorticography (ECoG) and of multi-lead depth electrode implantation known as SEEG (reviewed in Jerbi et al., 2009a). From the point of view of functional mapping, a major advantage of the multi-lead depth electrode implantation used in SEEG is the fact that the recordings are not limited to the cortical surface. An SEEG electrode consists of upto 15 contacts that probe multiple sites from lateral structures all the way through to medial wall regions (see
Figure 1A). This represents a major advantage when it comes to the investigation of DMN structures such as PCC, MPFC that are rarely probed by other electrophysiological techniques. Nevertheless, ECoG does occasionally involve placement of electrode strips on the surface of the medial wall and could in these cases be used for DMN investigations. Previous ECoG findings point toward SCP and gamma-range power as two types of electrical signals that display correlation patterns that mirror those observed in spontaneous fMRI BOLD signals (He et al., 2008; He and Raichle, 2009).

\section{DETECTION OF TASK-RELATED NEURAL DEACTIVATION WITH SEEG}

Given that a defining property of DMN is its task-related deactivation (i.e., negative BOLD response) during exteroceptive goal-directed behavior, the natural question that comes to mind is whether the DMN also displays task-related deactivations detectable in the electrophysiological signal. Robust SEEG deactivations in such regions may represent a putative neural correlate of task-related BOLD deactivations.

In the following, we further make the case for SEEG recordings as a particularly promising approach to study DMN deactivation, by providing samples of direct recordings from two regions of the
A

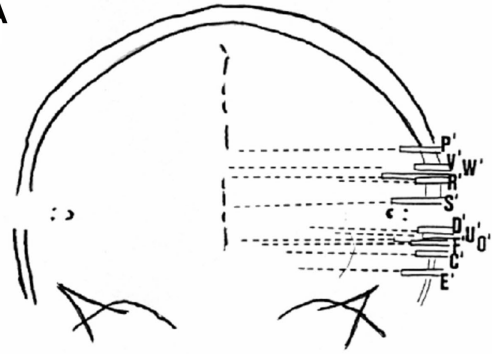

B Reading Task

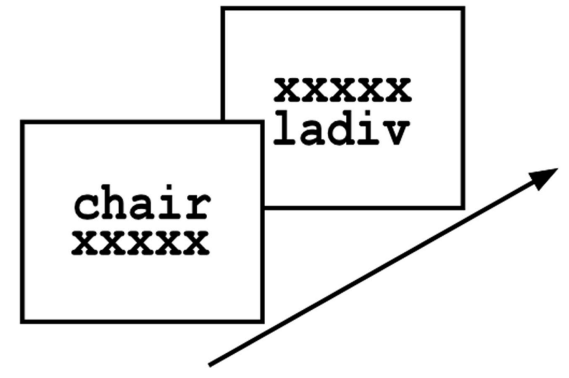

FIGURE 1 | Intracerebral stereotactic-EEG (SEEG) setting and cognitive paradigms. (A) SEEG recording procedure showing a typical implantation sketch based on a post-implantation X-ray scan (left panel) and a typical SEEG depth electrode array (right panel). See Materials and Methods in Supplementary Material for a detailed description of SEEG data acquisition. (B) Reading task (left panel): subjects were presented with stimuli that consisted of words and pseudowords. To ensure both categories were read, the subjects had to indicate whether the word represented a living or non-living item and, in the case of a pseudoword, whether it was made up of two syllables or

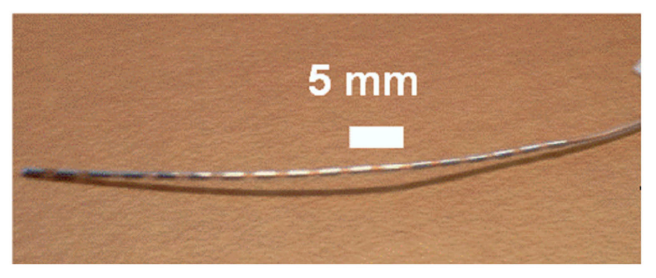

Navon Task

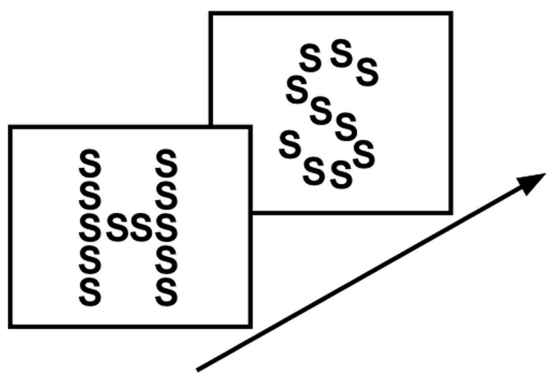

not. Navon task (right panel): subjects were presented with a large letter ("global letter"), which was itself composed of repeated smaller letters ("local letter"). The global and local letters could be either the letter "H" or the letter " $\mathrm{S}$," leading to four types of global-to-local stimulus configurations. At the beginning of a trial the subjects were cued to focus either on the global or the local level of the upcoming stimulus. The task consisted in identifying, as fast as possible, the stimulus letter at the level indicated by the cue. In both experiments the stimuli were presented for a maximum of $3 \mathrm{~s}$ and disappeared as soon as the subject responded. 
human DMN: the posterior cingulate cortex (PCC) and the MPFC. We then report, for the sake of comparison, electrode data acquired in the same subjects but from sites not assumed to be part of the DMN. The data presented here were acquired from subjects with SEEG depth electrodes implanted at multiple locations of the brain as part of their pre-surgical evaluation period (see supplementary material for details of the experimental procedures). The subjects participated in routine localizer experiments including a series of attention-demanding tasks such as a classical "global versus local" attention task (Navon, 1977) and a "word versus pseudoword" reading task (Figure 1B). Subsequent data analysis was strictly restricted to recording sites that showed no pathological activity. Figures 2 and 3 show results obtained with data recorded directly in PCC and MPFC respectively. Using time-frequency analysis of bipolar

A

Posterior Cingulate Cortex (PCC)
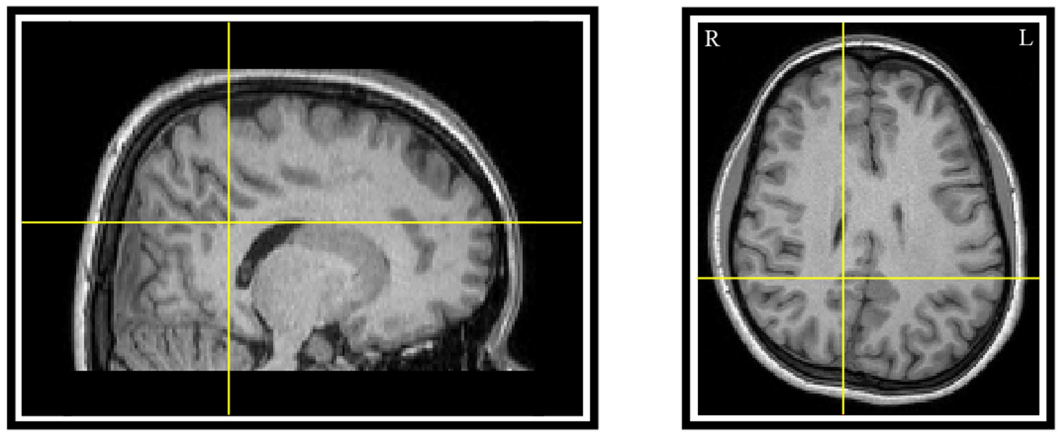

B

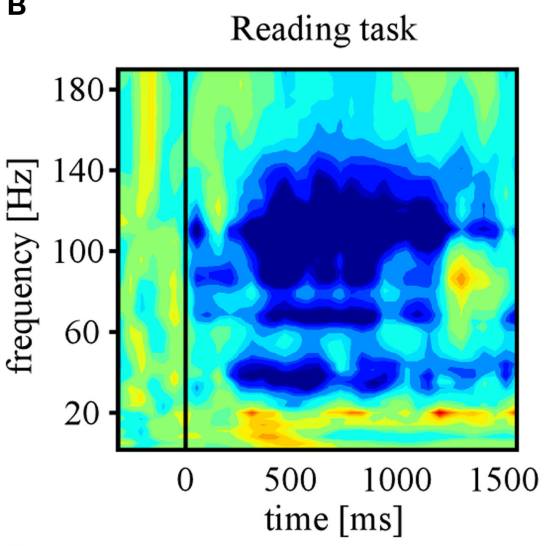

Z

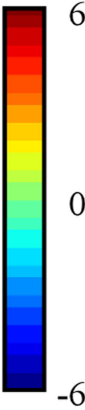

6

Navon task
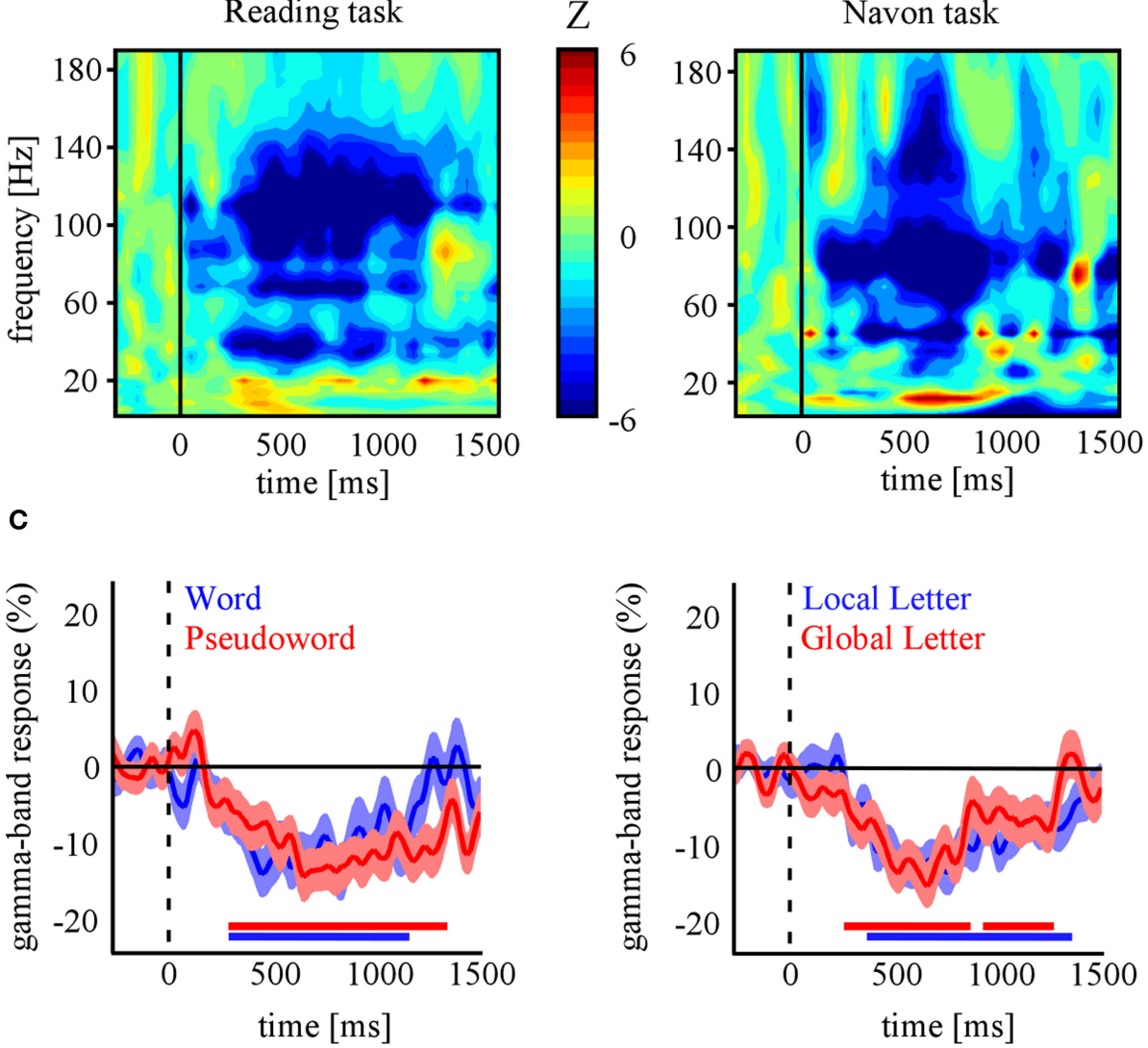

FIGURE 2 |Task-related gamma-band power suppressions in posterior cingulate cortex (PCC). (A) Anatomical location of the SEEG recording site in PCC of subject 1 (Talairach coordinates: $x=10, y=-38, z=35$ ). (B) Timefrequency representations of PCC activity for the reading (left panel) and Navon (right panel) tasks. Values represent task-related power modulations across time and frequency, compared with average baseline activity during fixation (Wilcoxon test). In both tasks strong decreases in PCC gamma power were

found (indicated by negative $Z$ values). (C) Time profile of percent power decreases (below baseline levels) at this electrode site for the conditions of each task (Left: Reading, Right: Navon). All conditions show significant gamma suppression in this region of DMN. The red/blue horizontal lines indicate statistical significance $(p<0.05)$ based on a Wilcoxon signed rank test and confidence intervals represent \pm s.e.m. (See Materials and Methods in Supplementary Material for more details). 

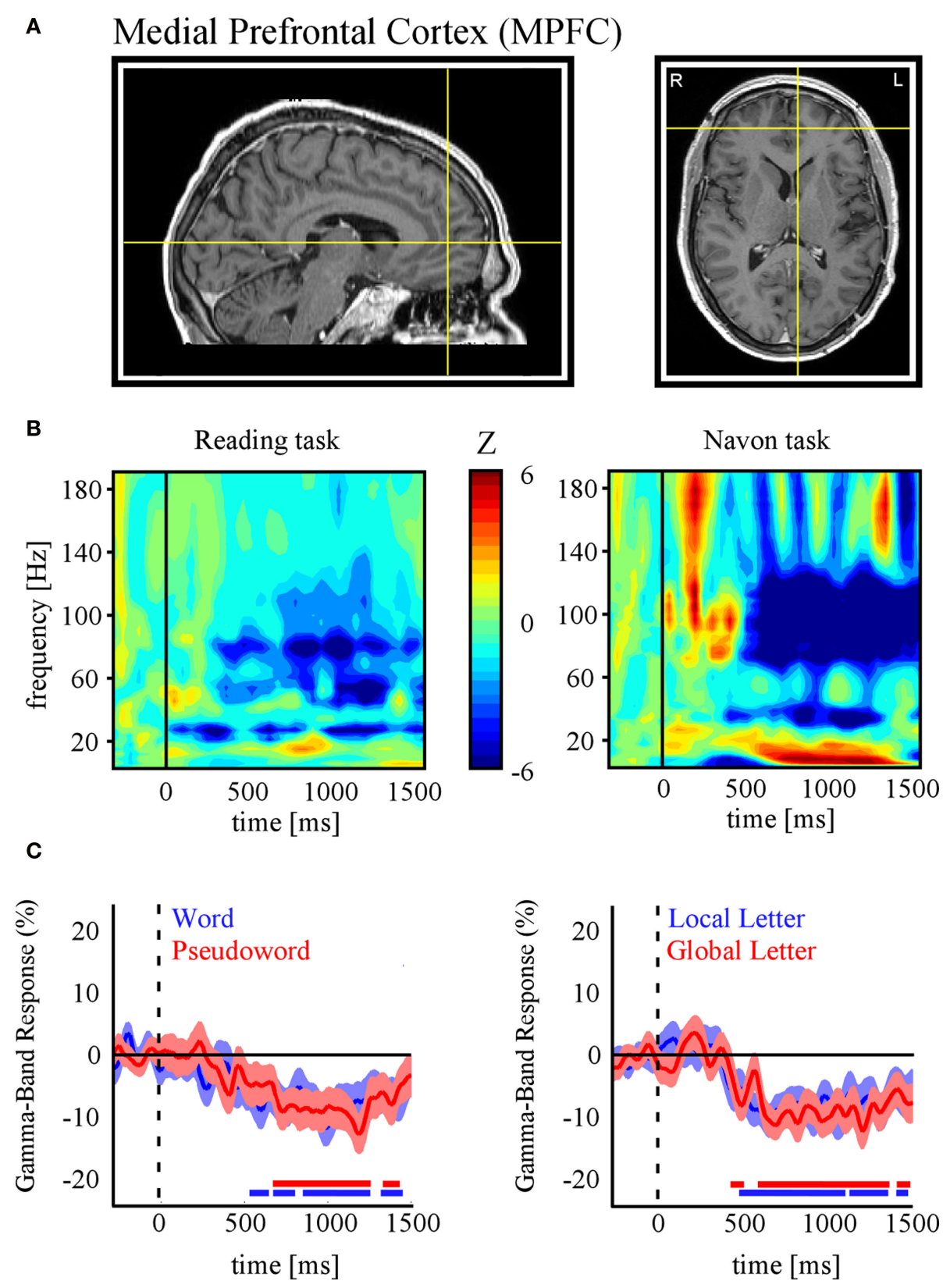

FIGURE 3 |Task-related gamma-band power suppressions in Medial Prefrontal Cortex (MPFC). (A) Anatomical location of the SEEG recording site in MPFC of subject 2 (Talairach coordinates: $x=-4, y=-46, z=-3$ ).

(B) Time-frequency representations of MPFC activity for the reading (left panel) and Navon (right panel) tasks. Strong decreases in MPFC gamma power were

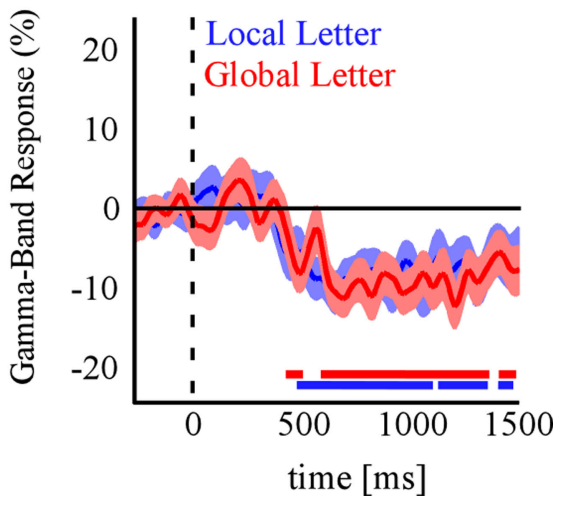

found in both tasks. (C) Time profile of percent power decreases (below baseline levels) at this electrode site for the conditions of each task (Left: Reading, Right: Navon). As for PCC (Figure 2), gamma activity in MPFC is significantly suppressed for all conditions. Display conventions and methods used are identical to those of Figure.2.

recordings in these areas we derive task-related maps that depict modulations of power across time and frequency, as compared to baseline levels (methods as in Jerbi et al., 2009a). Strikingly, compared to pre-stimulus baseline levels (during which subject simply fixate a cross), the Reading and the Navon tasks were associated with strong suppressions of power in the high gamma $(\sim 50-150 \mathrm{~Hz})$ observed in both PCC and MPFC sites (Figures 2B and 3B). Most importantly, as shown in Figures $2 \mathrm{C}$ and $3 \mathrm{C}$, the gamma-band deactivations were systematically present for all experimental con- ditions in both tasks. In addition to this task non-specificity, the fact that these intracranially recorded gamma suppressions occur in two regions known to be part of the DMN is in agreement with a putative link between SEEG gamma power deactivation and BOLD deactivation reported in the human fMRI DMN literature (Raichle et al., 2001). Importantly, this view is further supported by the fact that task-related gamma suppressions were not ubiquitous across recording sites. Applying the same spectral analysis to the data acquired in the same subjects but at other recording sites which 
are not part of DMN shows the inverse effect, i.e., task-related increases in the gamma-range and in both experiments (Figure 4). This was the case for recording sites in the fusiform gyrus (S1) and in the insula (S2). Moreover, it is noteworthy that the time course of gamma power suppression (Figures 2C and 3C) suggests that significant gamma-band deactivation starts on average around $250 \mathrm{~ms}$ in the PCC and then around $500 \mathrm{~ms}$ in MPFC. The deactivations are sustained in time lasting beyond $1000 \mathrm{~ms}$ post stimulus presentation. However, the data presented here are based only on two subjects. Clearly, more subjects will be needed to reliably estimate the temporal dynamics of gamma suppression and its relationship to behavior.

\section{DISCUSSION AND PERSPECTIVES}

A number of studies have established a tight relationship between BOLD activations and task-related increases in the gamma-range of the LFP signal in the same areas (Logothetis et al., 2001; Mukamel et al., 2005; Niessing et al., 2005; Lachaux et al., 2007a; Nir et al., 2007). Such observations lead to the corollary prediction that negative BOLD activity may also be correlated with gamma-band power suppressions. This has been shown to be indeed the case in mon- key primary visual area (V1) (Shmuel et al., 2006). Task-related decreases in high gamma power have also been reported with intracerebral recordings in human V1 during processing of complex visual stimuli (Lachaux et al., 2005). More generally, it is tempting to ask whether the so-called task-positive networks and task-negative networks revealed by the fMRI literature (Fox et al., 2005), are spatially coincident with task-related gamma power enhancement networks and task-related gamma power suppression networks respectively. This view implies that DMN areas would exhibit less gamma power during execution of attention-demanding tasks than during resting baseline periods. Support for this hypothesis has been reported in monkey PCC (Hayden et al., 2009). However, so far, equivalent findings in humans have been scarce. The frequency range of the high gamma-band $(\sim 40-160 \mathrm{~Hz})$ falls beyond the reach of most EEG studies that have been performed so far with the aim to assess the neural correlates of the DMN (e.g., Laufs et al., 2003; Mantini et al., 2007). This limitation, as well as source localization uncertainty (i.e., limited spatial resolution), can be in part overcome by the high signal-to-noise ratio and spatio-temporal resolution of intracerebral recordings. Although there have been a few reports of task-related gamma deactivations in some specific components of
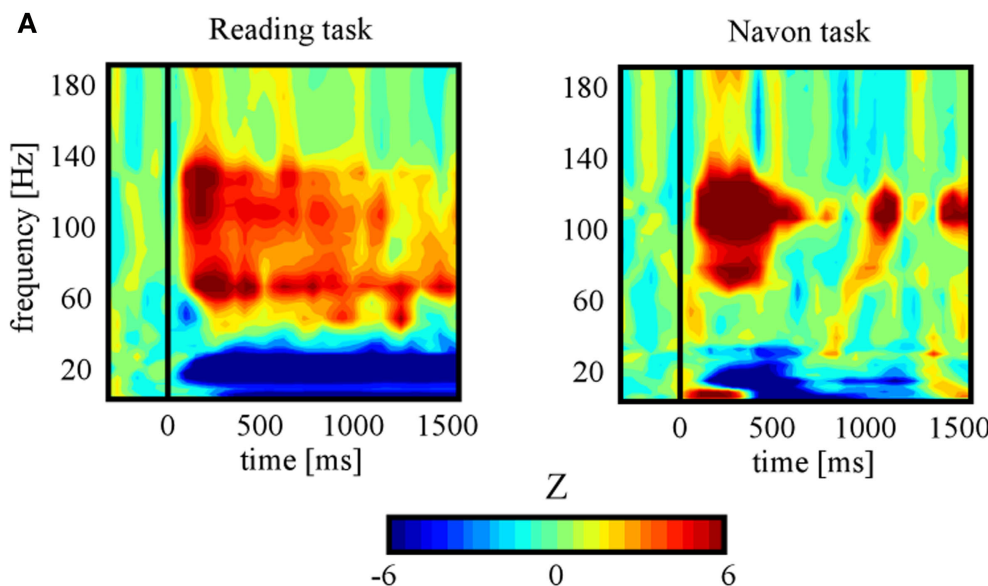

B

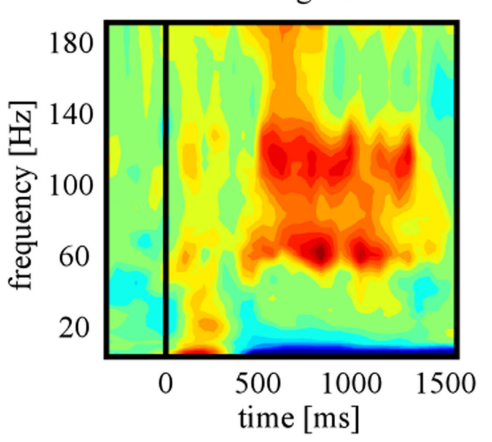

Navon task

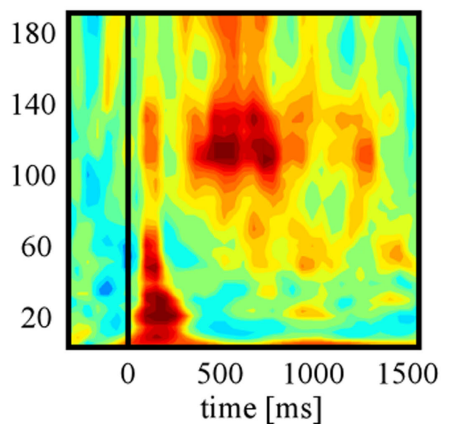

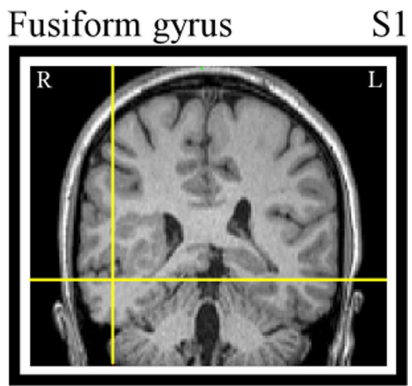

Insula

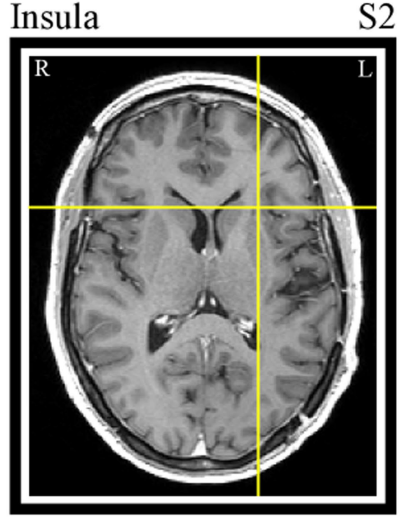

FIGURE 4 | Task-related gamma-band power increases. (A) Subject 1 : time-frequency representations during Reading (condition: pseudoword) (left panel) and Navon (condition: local) (central panel) tasks for an electrode located in the fusiform gyrus (right panel, Tailarach coordinates: $x=46, y=-43, z=-13$ ). Strong increases in gamma power were found in both tasks, in contrast to the decreases found for the same subject in PCC (see Figure 2). (B) Subject 2: time-frequency representations during Reading (condition: pseudoword) (left panel) and Navon (condition: local) (central panel) tasks for an electrode located in the fusiform gyrus (right panel, Tailarach coordinates: $x=-26, y=16, z=8$ ). The task-related enhancement of gamma power found here in both tasks is concurrent with task-related suppression in MPFC in the same subject (see Figure 3). Note that the full temporal profile of task-related gamma increases for the two conditions of each task for both subjects is provided in Figure $\mathbf{S 1}$ in Supplementary Material. 
human DMN using intracerebral recordings (Lachaux et al., 2008; Miller et al., 2009; Jung et al., 2010), an exhaustive investigation of all DMN structures and their fine-temporal dynamics using such techniques is hard to achieve and is still lacking.

The SEEG data presented here provides evidence for suppression of high-frequency activity in the human PCC and MPFC during task-engagement. This gamma-band deactivation $(40-150 \mathrm{~Hz})$ was task-related and occurred systematically across all experimental conditions. It is noteworthy that the Navon task we implemented (local versus global visual processing) induced significant gamma power suppressions in PCC, a region that has previously been shown to display negative BOLD in responses to the same paradigm performed with fMRI (Weissman et al., 2006). Remarkably, the high gamma suppression, found in the DMN, co-occurred with task-related enhancement outside the DMN (Figure 4). Elevated gamma power in the fusiform gyrus and in the anterior insula may reflect visual processing of the stimulus and intrinsic alertness activity respectively. Interestingly, increases in anterior insular gamma activity could be related to its role as part of the putative core taskset system (Dosenbach et al., 2006). Our observation of concurrent positive and negative high gamma responses, outside and inside the DMN respectively, is in line with the hypothesis that gamma modulations represent an electrical correlate of BOLD signal modulations. Critically, the population-level deactivation presented here extends a number of electrophysiological studies of DMN deactivation (e.g., Hayden et al., 2009; Miller et al., 2009) and strongly argues against the DMN being an epiphenomenon (Birn et al., 2008). Further studies across large populations of implanted patients are needed to strengthen and fine-tune these physiological interpretations. The illustrative data we report in PCC and MPFC highlight the potential of SEEG recordings as a tool to investigate the neurophysiology of DMN, and more generally speaking, of the resting state networks. Our group is actively pursuing the detection of brain-wide spatial distributions of gamma power decreases and increases in attentiondemanding tasks as well as the investigation of correlation patterns within the involved networks (Ossandon et al., 2009).

More generally, if we assume that broad-band gamma power suppressions observed in the default-mode areas reflect de facto neural disengagement, then we should also expect a concurrent reduction in local neuronal firing. An assessment of this hypothesis in the light of the tight relationship between spiking activity and broad-band gamma (Mukamel et al., 2005; Niessing et al., 2005; Manning et al., 2009; Whittingstall and Logothetis, 2009), leads to the hypothesis that default-mode areas may be characterized by task-related suppression of neuronal firing during attentive states. Although there is some recent evidence for this in monkey PCC (Hayden et al., 2009), little is known about task-related modulations of spiking activity specifically in default-mode structures of the human brain. This is primarily due to the rarity of unit recordings in human cortex and may change in the future if microelectrode recordings are used more often in clinical settings to probe DMN structures. Until then, various hypotheses about spike firing-rate modulations in human DMN may be inferred indirectly from the analysis of the broad-band gamma-range component of the EEG.

Furthermore, combining fMRI and EEG in simultaneous recordings will undoubtedly continue to provide unique insights into the links between electrophysiological and BOLD signals in resting state networks (Debener et al., 2005; Mantini et al., 2007; Scheeringa et al., 2008). The advent of simultaneous fMRI and intracranial EEG in the near future will move the multimodal investigations in this field a major step forward (Carmichael et al., 2010). However, the important impact that intracerebral recordings are expected to have on the study of the electrophysiological correlates of the DMN does not lessen the need for non-invasive electrophysiological approaches for this endeavor. Indeed, the precision of EEG and MEG will continue to improve thanks to the use of advanced source localization techniques (e.g., Baillet et al., 2001; Gross et al., 2001; Dalal et al., 2008) and signal decomposition tools such as independent component analysis (Mantini et al., 2007). Besides, given the putatively prominent role of high-frequency activity, improving the sensitivity of surface recordings to high gamma activity will be a critical issue (Jerbi et al., 2009a). Results of our recent study using simultaneously acquired MEG and intracerebral EEG data suggest that source imaging can indeed enhance our ability to detect the cortical generator of gamma activity with MEG (Dalal et al., 2009). In addition, several studies have shown that EEG signals can be contaminated by signals in the gamma-range that originate from eye muscles rather than cortical tissue (Reva and Aftanas, 2004; Trujillo et al., 2005; Yuval-Greenberg et al., 2008). Therefore, ruling out the effect of such saccade-related artifacts is a prerequisite for a reliable assessment of cortical gamma-band power using noninvasive techniques and constitutes an important topic for future research. As a matter of fact, we have recently shown that gammarange saccadic artifacts might, in some cases, even contaminate intracranial EEG recordings (Jerbi et al., 2009b).

Investigating the connectivity properties of intrinsic brain networks is clearly a topic where the input from electrophysiological recordings will be critical. Correlation and anti-correlation phenomena appear to be fundamental concepts surrounding resting state networks (Fox et al., 2005). Much still needs to be learned about how connectivity properties revealed with fMRI relate to brain-wide neural interactions revealed by MEG, EEG, and iEEG. Slow fluctuations in baseline activity observed with fMRI may be indirectly linked to higher frequency amplitude modulations via slow-to-fast cross-frequency interactions (Jensen and Colgin, 2007). Previous studies have shown that SCP can modulate higher frequency EEG activity (Vanhatalo et al., 2004), but also behavioral performance (Birbaumer et al., 1990; He et al., 2008; He and Raichle, 2009). Interestingly, in a recent study, Monto et al. (2008) used infra-slow EEG to provide evidence for very slow EEG fluctuations $(\sim 0.01 \mathrm{~Hz})$ that were correlated with slow perceptual performance modulations. The authors reported phase-amplitude coupling between these slow fluctuations and patterns of faster cortical oscillations. The use of within and cross-frequency coupling measures to assess local and long-range interactions in scalp-EEG, MEG, and intracranial EEG data is a rapidly growing field of research, yet its potential contribution to understanding the mechanisms of DMN is still largely underexploited.

Real-time monitoring of the electrophysiological activity within the DMN may open up the exciting perspective of performing online monitoring of vigilance or attention. What's more, realtime monitoring of DMN neuronal populations may allow for novel experimental designs with stimulation parameters that adapt online to the subjects state. While several challenges still 
need to be dealt with in order to achieve this with non-invasive measurements, real-time monitoring of high gamma activity in humans is readily achieved using depth SEEG recordings. We have implemented an online system for the estimation and visualization of power modulations in various frequency bands (including the high gamma-band) in conjunction with depth recording in epilepsy patients (Lachaux et al., 2007b). In addition to performing online functional mapping, this interface (dubbed Brain TV) could be seen as a window to the patient's ongoing and spontaneous brain activity. Therefore, with electrodes implanted in DMN areas, the Brain TV set-up could be used to monitor real-time modulations of power across the EEG spectrum during various states such as mind wandering or focused attention. Besides, the online monitoring of DMN activity could be beneficial to investigations into the functional role of DMN. For example, it may be possible to define online the timing of target or distractor stimulus presentation to correspond to specific states of the DMN. Ultimately, performing attention monitoring in real-time and non-invasively could have

\section{REFERENCES}

Anticevic, A., Repovs, G., Shulman, G. L., and Barch, D. M. (2010). When less is more: TPJ and default network deactivation during encoding predicts working memory performance. Neuroimage 49, 2638-2648.

Baillet, S., Mosher, J. C., and Leahy, R. M. (2001). Electromagnetic brain mapping. IEEE Signal Process. Mag. 18, $14-30$.

Ball, T., Demandt, E., Mutschler, I., Neitzel, E., Mehring, C., Vogt, K., Aertsen, A., and Schulze-Bonhage, A. (2008). Movement related activity in the high gamma range of the human EEG. Neuroimage 41, 302-310.

Birbaumer, N., Elbert, T., Canavan, A. G., and Rockstroh, B. (1990). Slow potentials of the cerebral cortex and behavior. Physiol. Rev. 70, 1-41.

Birn, R. M., Murphy, K., and Bandettini, P. A. (2008). The effect of respiration variations on independent component analysis results of resting state functional connectivity. Hum. Brain Mapp. $29,740-750$

Broyd, S. J., Demanuele, C., Debener, S., Helps, S. K., James, C. J., and SonugaBarke, E. J. (2009). Default-mode brain dysfunction in mental disorders: a systematic review. Neurosci. Biobehav. Rev. 33, 279-296.

Carmichael, D. W., Thornton, J. S., Rodionov, R., Thornton, R., McEvoy, A. W., Ordidge, R. J., Allen, P. J., and Lemieux, L. (2010). Feasibility of simultaneous intracranial EEG-fMRI in humans: a safety study. Neuroimage 49, 379-390.

Chen A. C., Feng, W., Zhao, H., Yin, Y., and Wang, P. (2008). EEG default mode network in the human brain: spectral regional field powers. Neuroimage 41, 561-574.
Cheyne, D., Bells, S., Ferrari, P., Gaetz, W., and Bostan, A. C. (2008). Selfpaced movements induce highfrequency gamma oscillations in primary motor cortex. Neuroimage 42, 332-342.

Corbetta, M., and Shulman, G. L. (2002). Control of goal-directed and stimulusdriven attention in the brain. Nat. Rev. Neurosci. 3, 201-215.

Crone, N. E., Sinai, A., and Korzeniewska, A. (2006). High-frequency gamma oscillations and human brain mapping with electrocorticography. Prog. Brain Res. 159, 275-295.

Dalal, S. S., Baillet, S., Adam, C., Ducorps, A., Schwartz, D., Jerbi, K., Bertrand, O., Garnero, L., Martinerie, J., and Lachaux, J.-P. (2009). Simultaneous MEG and intracranial EEG recordings during attentive reading. Neuroimage 45, 1289-1304.

Dalal, S. S., Guggisberg, A. G., Edwards, E., Sekihara, K., Findlay, A. M., Canolty, R. T., Berger, M.S., Knight, R. T., Barbaro, N. M., Kirsch, H. E., and Nagarajan, S. S. (2008). Five-dimensional neuroimaging: localization of the time-frequency dynamics of cortical activity. Neuroimage 40, 1686-1700.

Debener, S., Ullsperger, M., Siegel, M., Fiehler, K., von Cramon, D. Y., and Engel, A. K. (2005). Trial-by-trial coupling of concurrent electroencephalogram and functional magnetic resonance imaging identifies the dynamics of performance monitoring. J. Neurosci. 25, 11730-11737.

Dosenbach, N. U., Visscher, K. M., Palmer, E. D., Miezin, F. M., Wenger, K. K., Kang. H. C., Burgund, E. D., Grimes, A. L., Schlaggar, B. L., and Petersen, S. E. (2006). A core system for the implementation of task sets. Neuron 50, 799-812.

numerous clinical applications. Overall, a better understanding of the neural underpinnings and correlation dynamics within the DMN and, more globally, within resting state networks could have strong implications on the development of novel diagnostic and rehabilitation solutions for numerous neurological impairments.

\section{ACKNOWLEDGMENTS}

Support provided in part by the Fondation pour la Recherche Médicale (FRM) to Karim Jerbi, BrainSync FP7 European Project (Grant HEALTH-F2-2008-200728) to Juan R. Vidal, Marie Curie Fellowship (FP7-221097) from the European Commission to Sarang S. Dalal, and a doctoral fellowship from the Ministère de l'Education Nationale et la Recherche (France) to Tomas Ossandon.

\section{SUPPLEMENTARY MATERIAL}

The Supplementary Material for this article can be found online at http://www.frontiersin.org/neuroscience/systemsneuroscience/ paper/10.3389/fnsys.2010.00027/

Engel, A. K., Moll, C. K., Fried, I., and Ojemann, G. A. (2005). Invasive recordings from the human brain clinical insights and beyond. Nat. Rev Neurosci. 6, 35-47.

Fox, M. D., Snyder, A. Z., Vincent, J. L., Corbetta, M., Van Essen, D. C., and Raichle, M. E. (2005). The human brain is intrinsically organized into dynamic, anticorrelated functional networks. Proc. Natl. Acad. Sci. U.S.A. 102, 9673-9678.

Goldman, R. I., Stern, J. M., Engel, J. Jr., and Cohen,M.S.(2002).Simultaneous EEG and AMRI of the alpha rhythm. Neuroreport 13, 2487-2492.

Greicius, M. (2008). Resting-state functional connectivity in neuropsychiatric disorders. Curr. Opin. Neurol. 21 424-430.

Greicius, M. D., Kiviniemi, V., Tervonen, O., Vainionpaa, V., Alahuhta, S., Reiss, A. L., and Menon, V. (2008). Persistent default-mode network connectivity during light sedation. Hum. Brain Mapp. 29, 839-847.

Greicius, M. D., Krasnow, B., Reiss, A. L., and Menon, V. (2003). Functional connectivity in the resting brain: a network analysis of the default mode hypothesis. Proc. Natl. Acad.Sci. U.S.A. 100, 253-258.

Gross, J., Kujala, J., Hamalainen, M. Timmermann, L., Schnitzler, A., and Salmelin, R. (2001). Dynamic imaging of coherent sources: studying neural interactions in the human brain. Proc. Natl. Acad. Sci. U.S.A. 98, 694-699.

Gusnard, D.A.,Akbudak, E., Shulman, G. L., and Raichle, M. E. (2001). Medial prefrontal cortex and self-referential mental activity: relation to a default mode of brain function. Proc. Natl. Acad. Sci. U.S.A. 98, 4259-4264.
Gusnard, D. A., and Raichle, M.E. (2001). Searching for a baseline: functional imaging and the resting human brain Nat. Rev. Neurosci. 2, 685-694.

Hayden, B. Y., Smith, D. V., and Platt, M. L. (2009). Electrophysiological correlates of default-mode processing in macaque posterior cingulate cortex. Proc. Natl. Acad. Sci. U.S.A. 106, 5948-5953.

He, B. J., and Raichle, M. E. (2009). The fMRI signal, slow cortical potential and consciousness. Trends Cogn. Sci. (Regul. Ed.) 13, 302-309.

He, B. J., Snyder, A. Z., Zempel, J. M., Smyth, M. D., and Raichle, M. E. (2008). Electrophysiological correlates of the brain's intrinsic large-scale functional architecture. Proc. Natl. Acad. Sci. U.S.A. 105, 16039-16044.

Jann, K., Dierks, T., Boesch, C., Kottlow, M., Strik, W., and Koenig, T. (2009). BOLD correlates of EEG alpha phaselocking and the fMRI default mode network. Neuroimage 45, 903-916.

Jensen, O., and Colgin, L. L. (2007). Crossfrequency coupling between neuronal oscillations. Trends Cogn. Sci. (Regul. Ed.) 11, 267-269.

Jensen, O., Kaiser, J., and Lachaux, J. P. (2007). Human gamma-frequency oscillations associated with attention and memory. Trends Neurosci. 30, 317-324.

Jerbi, K., Ossandon, T., Hamame, C. M., Senova, S., Dalal, S. S., Jung, J., Minotti, L., Bertrand, O., Berthoz, A., Kahane, P., and Lachaux, J. P. (2009a). Task-related gamma-band dynamics from an intracerebral perspective: review and implications for surface EEG and MEG. Hum. Brain Mapp. 30, 1758-1771.

Jerbi, K., Freyermuth, S., Dalal, S., Kahane, P., Bertrand, O., Berthoz, A., and 
Lachaux, J. P. (2009b). Saccade related gamma-band activity in intracerebral EEG: dissociating neural from ocular muscle activity. Brain Topogr. 22, 18-23.

Jung, J., Jerbi, K., Ossandon, T., Ryvlin, P., Isnard, J., Bertrand, O., Guénot, M., Mauguière, F., and Lachaux, J. P. (2010). Brain responses to success and failure: direct recordings from human cerebral cortex. Hum. Brain Mapp. (in press) doi: 10.1002/hbm.20930.

Kahane, P., Landre, E., Minotti, L., Francione, S., and Ryvlin, P. (2006). The Bancaud and Talairach view on the epileptogenic zone: a working hypothesis. Epileptic Disord. 8(Suppl. 2), S16-S26.

Kahane, P., Minotti, L., Hoffmann, D., Lachaux, J., and Ryvlin, P. (2004). "Invasive EEG in the definition of the seizure onset zone: depth electrodes," in Handbook of Clinical Neurophysiology. Pre-Surgical Assessment of the Epilepsies with Clinical Neurophysiology and Functional Neuroimaging, eds F. Rosenow and H. O. Lüders, (Amsterdam, Boston: Elsevier Science), 109-133.

Khader, P., Schicke, T., Röder, B., and Rösler, F. (2008). On the relationship between slow cortical potentials, and BOLD signal changes in humans. Int. J. Psychophysiol. 67, 252-261.

Lachaux, J. P., Fonlupt, P., Kahane, P., Minotti, L., Hoffmann, D., Bertrand, O., and Baciu, M. (2007a). Relationship between task-related gamma oscillations and BOLD signal: new insights from combined $\mathrm{AMRI}$ and intracranial EEG. Hum. Brain Mapp. 28, 1368-1375.

Lachaux, J. P., Jerbi, K., Bertrand, O., Minotti,L.,Hoffmann,D.,Schoendorff, B., and Kahane, P. (2007b). A blueprint for real-time functional mapping via human intracranial recordings. PLoS ONE 2, e1094. doi: 10.1371/journal. pone.0001094.

Lachaux, J. P., George, N., Tallon-Baudry, C., Martinerie, J., Hugueville, L., Minotti, L., Kahane, P., and Renault, B. (2005). The many faces of the gamma band response to complex visual stimuli. Neuroimage 25, 491-501.

Lachaux, J. P., Jung, J., Mainy, N., Dreher, J. C., Bertrand, O., Baciu, M., Minotti, L., Hoffmann, D., and Kahane, P. (2008). Silence is golden: transient neural deactivation in the prefrontal cortex during attentive reading. Cereb. Cortex 18, 443-450.

Laufs, H. (2008). Endogenous brain oscillations and related networks detected by surface EEG-combined fMRI. Hum. Brain Mapp. 29, 762-769.

Laufs, H., Krakow, K., Sterzer, P., Eger, E., Beyerle, A., Salek-Haddadi, A., and Kleinschmidt, A. (2003). Electroencephalographic signatures of attentional and cognitive default modes in spontaneous brain activity fluctuations at rest. Proc. Natl. Acad. Sci. U.S.A. 100, 11053-11058.

Logothetis, N. K., Pauls, J., Augath, M., Trinath, T., and Oeltermann,A. (2001). Neurophysiological investigation of the basis of the fMRI signal. Nature 412, 150-157.

Mainy, N., Jung, J., Baciu, M., Kahane, P., Schoendorff, B., Minotti, L., Hoffmann, D., Bertrand, O., and Lachaux, J. P. (2008). Cortical dynamics of word recognition. Hum. Brain Mapp. 29, 1215-1230.

Manning, J. R., Jacobs, J., Fried, I., and Kahana, M. J. (2009). Broadband shifts in local field potential power spectra are correlated with single-neuron spiking in humans. J. Neurosci. 29, 13613-13620.

Mantini, D., Perrucci, M. G., Del Gratta, C., Romani, G. L., and Corbetta, M. (2007). Electrophysiological signatures of resting state networks in the human brain. Proc. Natl. Acad. Sci. U.S.A. 104, 13170-13175.

Mason, M. F., Norton, M. I., Van Horn, J. D., Wegner, D. M., Grafton, S. T., and Macrae, C. N. (2007). Wandering minds: the default network and stimulus-independent thought. Science 315, 393-395.

Miller, K. J., Weaver, K. E., and Ojemann, J. G. (2009). Direct electrophysiological measurement of human default network areas. Proc. Natl. Acad. Sci. U.S.A. 106, 12174-12177.

Monto, S., Palva, S., Voipio, J., and Palva, J. M. (2008). Very slow EEG fluctuations predict the dynamics of stimulus detection and oscillation amplitudes in humans. J. Neurosci. 28, 8268-8272.

Moosmann, M., Ritter, P., Krastel, I., Brink, A., Thees, S., Blankenburg, F., Taskin, B., Obrig, H., and Villringer, A. (2003). Correlates of alpha rhythm in functional magnetic resonance imaging and near infrared spectroscopy. Neuroimage 20, 145-158.

Mukamel, R., Gelbard, H., Arieli, A., Hasson, U., Fried, I., and Malach, R. (2005). Coupling between neuronal firing, field potentials, and FMRI in human auditory cortex. Science 309, 951-954.

Navon, D. (1977). Forest before trees: the precedence of global features in visual perception. Cogn. Psychol. 9, 353-383.

Niessing, J., Ebisch, B., Schmidt, K. E., Niessing, M., Singer, W., and Galuske, R. A. (2005). Hemodynamic signals correlate tightly with synchronized gamma oscillations. Science 309, 948-951.

Nir, Y., Fisch, L., Mukamel, R., GelbardSagiv, H., Arieli, A., Fried, I., and Malach, R. (2007). Coupling between neuronal firing rate, gamma LFP, and BOLD fMRI is related to interneuronal correlations. Curr. Biol. 17, 1275-1285.

Ossandon, T., Jerbi, K., Bayle, D., Bertrand, O., Kahane, P., and Lachaux, J.-P. (2009). Task-related gamma band suppressions: a plausible electrophysiological correlate of the defaultmode network? Soc. Neurosci. Abstract 2009 (Program No 804.10) SfN 2009, Chicago, IL.

Pfurtscheller, G., and Cooper, R. (1975). Frequency dependence of the transmission of the EEG from cortex to scalp. Electroencephalogr. Clin. Neurophysiol. 38, 93-96.

Raichle, M. E., MacLeod, A. M., Snyder, A Z., Powers, W. J., Gusnard, D. A., and Shulman, G. L. (2001). A default mode of brain function. Proc. Natl. Acad. Sci. U.S.A. 98, 676-682.

Reva, N. V., and Aftanas, L. I. (2004). The coincidence between late nonphaselocked gamma synchronization response and saccadic eye movements. Int. J. Psychophysiol. 51, 215-222.

Scheeringa, R., Bastiaansen, M. C. Petersson, K. M., Oostenveld, R., Norris, D. G., and Hagoort, P. (2008). Frontal theta EEG activity correlates negatively with the default mode network in resting state. Int. J. Psychophysiol. 67, 242-251.

Shmuel, A., Augath, M., Oeltermann, A., and Logothetis, N. K. (2006). Negative functional MRI response correlates with decreases in neuronal activity in monkey visual area V1. Nat. Neurosci. 9, 569-577.

Shulman, G. L., Astafiev, S. V., McAvoy, M. P., d'Avossa, G., and Corbetta, M. (2007). Right TPJ deactivation during visual search: functional significance and support for a filter hypothesis. Cereb. Cortex 17, 2625-2633.

Tecchio, F., Zappasodi, F., Porcaro, C. Barbati, G., Assenza, G., Salustri, C., and Rossini, P. M. (2008). Highgamma band activity of primary hand cortical areas: a sensorimotor feedback efficiency index. Neuroimage 40, 256-264.

Trujillo, L. T., Peterson, M.A., Kaszniak, A W., and Allen, J. J. (2005). EEG phase synchrony differences across visual perception conditions may depend on recording and analysis methods. Clin. Neurophysiol. 116, 172-189.

Uddin, L. Q., Kelly, A. M., Biswal, B. B., Xavier Castellanos, F., and Milham, M. P. (2009). Functional connectivity of default mode network components: correlation, anticorrelation, and causality. Hum. Brain Mapp. 30, 625-637.

Van Der Werf, J., Jensen, O., Fries, P., and Medendorp, W. P. (2010). Neuronal synchronization in human posterior parietal cortex during reach planning. J. Neurosci. 30, 1402-1412.

Vanhatalo, S., Palva,J. M., Holmes, M. D., Miller, J. W., and Voipio, J, Kaila, K. (2004). Infraslow oscillations modulate excitability and interictal epileptic activity in the human cortex during sleep. Proc. Natl. Acad. Sci. U.S.A. 101, 5053-5057.

Vincent, J. L., Patel, G. H., Fox, M. D., Snyder, A. Z., Baker, J. T., Van Essen, D. C., Zempel, J. M., Snyder, L. H., Corbetta, M., and Raichle, M. E. (2007). Intrinsic functional architecture in the anaesthetized monkey brain. Nature 447, 83-86.

Waldert, S., Preissl, H., Demandt, E., Braun, C., Birbaumer, N., Aertsen, A., and Mehring, C. (2008). Hand movement direction decoded from MEG and EEG. J. Neurosci. 28, 1000-1008.

Weissman, D. H., Roberts, K. C., Visscher, K.M., and Woldorff,M.G. (2006). The neural bases of momentary lapses in attention. Nat. Neurosci. 9, 971-978.

Whittingstall, K., and Logothetis, N. K. (2009). Frequency-band coupling in surface EEG reflects spiking activity in monkey visual cortex. Neuron 64, 281-289.

Yuval-Greenberg, S., Tomer, O., Keren, A. S., Nelken, I., and Deouell, L. Y. (2008). Transient induced gammaband response in EEG as a manifestation of miniature saccades. Neuron 58, 429-441.

Zumer, J. M., Brookes, M. J., Stevenson, C. M., Francis, S. T., and Morris, P. G. (2010). Relating BOLD fMRI and neural oscillations through convolution and optimal linear weighting. Neuroimage 49, 1479-1489.

Conflict of Interest Statement: The authors declare that the research was conducted in the absence of any commercial or financial relationships that could be construed as a potential conflict of interest.

Received:09 February 2010; paperpending published: 28 March 2010; accepted:04 June 2010; published online: 28 June 2010.

Citation: Jerbi K, Vidal JR, Ossandon T, Dalal SS, Jung J, Hoffmann D, Minotti L, Bertrand O, Kahane P and Lachaux J-P (2010) Exploring the electrophysiological correlates of the default-mode network with intracerebral EEG. Front. Syst. Neurosci. 4:27. doi: 10.3389/fnsys.2010.00027

Copyright (C) 2010 Jerbi, Vidal, Ossandon, Dalal, Jung, Hoffmann, Minotti, Bertrand, Kahane and Lachaux. This is an openaccess article subject to an exclusive license agreement between the authors and the Frontiers Research Foundation, which permits unrestricted use, distribution, and reproduction in any medium, provided the original authors and source are credited. 